\title{
Disinfection of methicillin-resistant Staphylococcus aureus, vancomycin-resistant Enterococcus faecium and Acinetobacter baumannii using Klaran WD array system
}

\author{
Richard M. Mariita* and Rajul V. Randive
}

\begin{abstract}
Hospital-associated infections (HAls) are a major burden in healthcare systems. In this study, UVC LEDs emitting radiation from 260 to $270 \mathrm{~nm}$ were evaluated for effectiveness in reducing methicillin-resistant Staphylococcus aureus (MRSA), vancomycinresistant Enterococcus faecium and Acinetobacter baumannii. The array has four WD LEDs, each with $70 \mathrm{~mW}$ placed at $7 \mathrm{~cm}$ from test organisms. With $11.76 \mathrm{~mJ} \mathrm{~cm}^{-2}$, the study obtained $99.99 \%$ reduction ( $\log _{10}$ reduction factor of 4 ) against MRSA and VRE. For A. baumannii, $9 \mathrm{~m} \mathrm{~J} \mathrm{~cm} \mathrm{~cm}^{-2}$ obtained $99.999 \%$ reduction $\left(\log _{10}\right.$ reduction factor of 5). These results present scientific evidence on how effective UVC LEDs can be used in the fight against HAls.
\end{abstract}

\section{INTRODUCTION}

Worldwide, hospital-acquired infections (HAIs) are responsible for extended admissions, increased medical costs, and noticeable morbidity and mortality [1]. Per year, it is approximated that in the USA alone, 2 million patients suffer from HAIs, while nearly 90000 are estimated to die. The overall direct cost of HAIs to hospitals ranges from US \$28-45 billion [2]. The micro-organisms responsible for HAIs include methicillin-resistant Staphylococcus aureus (MRSA), vancomycin-resistant enterococci (VRE) and Acinetobacter baumannii. Here, UVC LEDs emitting radiation at 260-270 $\mathrm{nm}$ were evaluated for effectiveness in reducing MRSA ATCC 33592, VRE ATCC 700221 and A. baumannii ATCC 19606.

S. aureus is a major pathogen both within hospitals and in the community, as healthcare systems in the USA and Europe have seen the prevalence of MRSA increase from $<3 \%$ in the early 1980s to rates as high as 40\% in the 1990s [3]. In 2017 alone, the USA had 119000 infections and almost 20000 deaths [4]. MRSA costs $\sim$ US $\$ 10$ billion a year to treat in the USA, averaging US \$60000-70000 per patient [5]. MRSA can be transmitted via surgical tools, high-touch surfaces, air in surgical units as well via intubation in adult intensive care unit (ICU), causing pneumonia [3].
VRE is of medical importance, as it is associated with serious multidrug-resistant infections [6]. Moreover, in the USA, the emergence of VRE in water is threatening the health of human beings due to antibiotic resistance [6] and the life-threatening nature of some VRE infections [6]. VRE has been isolated from most sites and objects in healthcare facilities, including medical equipment (ventilator tubing, pumps, wash bowls, automated medication dispensers, intravenous poles), monitoring devices (call bells, electrocardiographic monitors, pulse oximeters, glucose meters, stethoscopes, electronic thermometers, blood pressure cuffs, keyboards, wall-mounted control panels), furniture (telephones, air cushions, headboards, tables, chairs, bed rails), toilet seats, doors, floors and linens [7]. In the USA, hospital costs due to VRE infections vary between US \$9949-77558 [8].

Globally, A. baumannii is one of the most clinically significant multidrug-resistant organisms [9]. A. baumannii can be aerosolized, and has been found to contaminate trauma ICUs and HVAC systems, especially in locations with high humidity, causing pneumonia and urinary tract and bloodstream infections [9]. In 2017, carbapenem-resistant Acinetobacter caused an estimated 8500 infections in hospitalized patients and 700 estimated deaths in the USA alone [10] with US \$281 million in healthcare costs. There are few treatment options, hence the

Received 14 January 2021; Accepted 26 July 2021; Published 15 September 2021

Author affiliations: ${ }^{1}$ Crystal IS, Inc., an Asahi Kasei company, 70 Cohoes Avenue, Green Island, New York, 12183, USA.

*Correspondence: Richard M. Mariita, richard.mariita@cisuvc.com

Keywords: Acinetobacter baumannii; disinfection; healthcare-associated infections; methicillin-resistant Staphylococcus aureus (MRSA); UVC LEDs;

vancomycin-resistant enterococcus faecium; HVAC.

Abbreviations: HAls, hospital-acquired infections; HVAC, heating, ventilation, and air conditioning; LEDs, light emitting diodes; MRSA, methicillin-

resistant Staphylococcus aureus; UVC, ultraviolet-C; VRE, vancomycin-resistant enterococci.

$000194 \odot 2021$ The Authors 
Table 1. Effectiveness of UVC disinfection against methicillin-resistant Staphylococcus aureus (MRSA), vancomycin-resistant Enterococcus faecium and Acinetobacter baumannii at $7 \mathrm{~cm}$ using an array with 4 UVC LEDs. Experiments were performed in triplicate and data are expressed as means

\begin{tabular}{|c|c|c|c|c|c|}
\hline Strain & UVC dose $\left(\mathrm{mJ} \mathrm{cm} \mathrm{cm}^{-2}\right)$ & Irradiation time (seconds) & Control c.f.u. $\mathrm{ml}^{-1}$ (-UVC) & Test c.f.u. ml $^{-1}$ (+UVC) & LRV \\
\hline VRE & 6.18 & 6 & $1.00 \mathrm{E}+09$ & $5.43 \mathrm{E}+06$ & 2.26 \\
\hline VRE & 9.00 & 9 & $1.00 \mathrm{E}+09$ & $6.67 \mathrm{E}+05$ & 3.18 \\
\hline VRE & 11.76 & 12 & $1.00 \mathrm{E}+09$ & $5.57 \mathrm{E}+04$ & 4.25 \\
\hline MRSA & 3.15 & 3 & $1.61 \mathrm{E}+08$ & $1.10 \mathrm{E}+07$ & 1.17 \\
\hline MRSA & 6.18 & 6 & $1.61 \mathrm{E}+08$ & $1.00 \mathrm{E}+06$ & 2.21 \\
\hline MRSA & 9.00 & 9 & $1.61 \mathrm{E}+08$ & $1.33 \mathrm{E}+05$ & 3.08 \\
\hline MRSA & 11.76 & 12 & $1.61 \mathrm{E}+08$ & $1.00 \mathrm{E}+04$ & 4.21 \\
\hline A. baumannii & 6.18 & 6 & $2.49 \mathrm{E}+07$ & $2.30 \mathrm{E}+02$ & 5.03 \\
\hline A. baumannii & 9.00 & 9 & $2.49 \mathrm{E}+07$ & $8.67 \mathrm{E}+01$ & 5.46 \\
\hline A. baumannii & 11.76 & 12 & $2.49 \mathrm{E}+07$ & $2.53 \mathrm{E}+01$ & 5.99 \\
\hline A. baumannii & 16.5 & 15 & $2.49 \mathrm{E}+07$ & $3.33 \mathrm{E}+00$ & 6.87 \\
\hline
\end{tabular}

designation of A. baumannii as a pathogen of urgent concern and priority [10].

\section{Study objectives}

Given the economic and health challenges associated with HAIs, additional effective strategies for disease prevention are needed. The objective of this study was to evaluate the disinfection performance of a UVC LED array against three causative agents responsible for HAIs. Specifically, the study investigated the UVC dose required for inactivation of MRSA (ATCC 33592), Enterococcus faecium (ATCC 700221) and A. baumannii (ATCC 19606). These results will be used as a baseline in determining the UVC dose required in the control of causative agents responsible for HAIs, thus minimizing extensive use of antibiotics.

\section{METHODS}

\section{Strain and culture conditions}

All strains were stored at $-80^{\circ} \mathrm{C}$ in appropriate culture media using sterile $20 \%$ glycerol stock. Working strain cultures were stored at $4{ }^{\circ} \mathrm{C}$ and propagated before being used in UVC disinfection tests. MRSA and A. baumannii strains were propagated in ATCC Medium 3 (nutrient agar/broth), whereas VRE was propagated in ATCC Medium 44 (brain heart infusion agar/broth). Cultures were incubated for 18-20 $\mathrm{h}$ at $37^{\circ} \mathrm{C}$ with shaking at 180 r.p.m. For use, each strain was harvested by centrifugation at 4000 r.p.m. for $10 \mathrm{~min}$. The pellets were washed using $1 \times$ phosphate-buffered saline (PBS) three times for $10 \mathrm{~min}$. Between each wash, the supernatant was discarded, and the remaining pellet was resuspended by vortexing. After being washed thrice, the pellet was resuspended in $1 \times$ PBS used for static dosing study. Agar plates containing irradiated strains were incubated at $37^{\circ} \mathrm{C}$ for a further 18-20 h. Static dosing was performed in three independent replicates and means were used in statistics.

\section{UVC LED disinfection}

The UVC disinfection efficiency of a 4 UVC LED array was evaluated using stationary growth phase bacteria. The array emitted radiation at a wavelength of $260-270 \mathrm{~nm}$, as confirmed using an Ocean Optics USB4000 photospectrometer. The distance between the UVC LEDs and agar plate inoculated with bacteria suspension was $7 \mathrm{~cm}$. Disinfection efficacy was assessed after 3, 6, 9, 12 and $15 \mathrm{~s}$ of irradiation (Table 1). Controls were not UVC dosed. Log reduction value (LRV) was calculated using the equation:

$$
\operatorname{LRV}=\log _{10}\left(\frac{\mathrm{A}}{\mathrm{B}}\right)
$$

where $A$ is c.f.u. $\mathrm{ml}^{-1}$ for $\mathrm{UVC}$ controls ( $-\mathrm{UVC}$ ) and $B$ is c.f.u. $\mathrm{ml}^{-1}$ for UVC dosed (UVC+).

To determine the relationship between irradiation time and disinfection efficacy against each test strain, linear regression analysis was performed using GraphPad software (https:// www.graphpad.com/).

\section{RESULTS}

Table 1 shows that the UVC LED array is very effective against the study test strains (MRSA ATCC 33592, VRE ATCC 700221 and A. baumannii ATCC 19606). The study revealed 


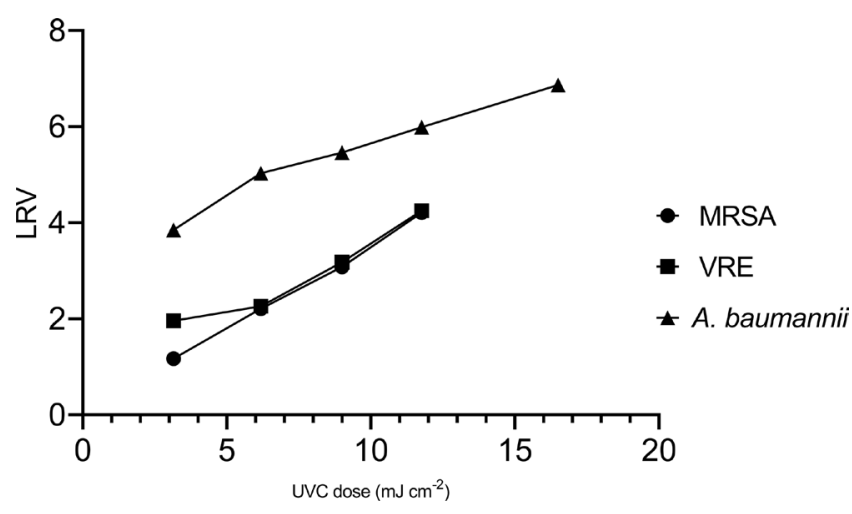

Fig. 1. Disinfection efficacy of UVC against key pathogens associated with HAls. A. baumannii was more sensitive against UVC compared to MRSA and VRE.

that 12 seconds of irradiation time yielded $11.76 \mathrm{~mJ} \mathrm{~cm}^{-2} \mathrm{UVC}$ dose, which was enough to obtain $>$ LRV4 (99.99\% reduction) against the three test strains (Table 1). Additionally, the study revealed that $A$. baumannii was more susceptible to UVC radiation than MRSA and VRE strains (Fig. 1).

Linear regression analysis at $95 \%$ for MRSA $\left(R^{2}=0.9969\right.$, $P=0.0016)$, A. baumannii $\left(R^{2}=0.9681, P=0.0024\right)$ and VRE $\left(R^{2}=0.9403, P=0.0303\right)$ displayed strong association between irradiation time and disinfection efficacy.

\section{DISCUSSION}

MRSA is transmitted through high-touch surfaces or direct contact with infected individuals [11]. The mode of transmission for A. baumannii [12] and VRE [13] is similar to that of MRSA. Disinfection of high-touch surfaces is thus an obvious intervention for infection control [14], especially in healthcare facilities to protect the already vulnerable patients. Although enhanced cleaning has been shown to reduce MRSA infections in wards [14], additional measures can be put in place in areas such as surgical rooms, where some MRSA transmission can be caused by airborne transmission [15].

This study has demonstrated in the laboratory that the use of LEDs that emit 260-270 $\mathrm{nm}$ UVC radiation has the capability to achieve high levels of inactivation of micro-organisms that are causative agents of HAIs. The UVC radiation acts by exerting germicidal affect on DNA, leading to breakage of molecular bonding [16]. A microbial disinfection strategy using UVC LEDs leaves no waste and, unlike mercury lamps, there is no potential exposure to mercury and there are no disposal-related hazards. The inactivation efficacy from our baseline study supports previous demonstrations that revealed that the use of UVC radiation will be a beneficial adjunct to other existing strategies, leading to further reduction of microbial agents responsible for HAIs [17].

Our study revealed that A. baumannii was more susceptible to UVC radiation than MRSA and VRE strains. This is possibly due to A. baumannii being Gram-negative [18] as opposed to MRSA and VRE, which are Gram-positive [19]. As a rule, Gram-positive bacteria are more resistant to UV compared to Gram-negative ones [20]. This can be attributed to morphological differences. For instance, the presence of thicker cell walls in Gram-positive bacteria could reduce the amount of UVC reaching the cellular DNA [21].

Based on these baseline findings, it is proposed that a further feasibility study in areas such as in intensive care and surgical units as well as other high-touch surfaces be undertaken.

\section{Conclusion}

The results from this study suggest that arrays of Klaran WD UVC LEDs emitting radiation between $260-270 \mathrm{~nm}$ can provide effective and rapid decontamination of HAIs. Further in situ tests to assess usability and relative performance would be justified.

Funding information

The authors received no specific grant from any funding agency.

Acknowledgements

The authors thank Michelle Lottridge, Amy Miller and Chris Scully for their technical help and review of manuscript.

Conflicts of interest

Both R. M. M. and R. V. R. work for Crystal IS, an Asahi Kasei company that manufactures UVC LEDs.

References

1. De Angelis G, Murthy A, Beyersmann J, Harbarth S. Estimating the impact of healthcare-associated infections on length of stay and costs. Clin Microbiol Infect 2010;16:1729-1735.

2. Stone PW. Economic burden of healthcare-associated infections: An american perspective. Expert Rev Pharmacoecon Outcomes Res 2009:9:417-422.

3. David MZ, Daum RS. Community-associated methicillin-resistant Staphylococcus aureus: epidemiology and clinical consequences of an emerging epidemic. Clin Microbiol Rev 2010;23:616-687.

4. Kavanagh KT. Control of MSSA and MRSA in the United States: protocols, policies, risk adjustment and excuses. Antimicrob Resist Infect Control 2019:8:1-8.

5. Valiquette L, Chakra CNA, Laupland KB. Financial impact of health care-associated infections: When money talks. Can J Infect Dis Med Microbiol 2014;25:71-74.

6. Ahmed MO, Baptiste KE. Vancomycin-resistant enterococci: a review of antimicrobial resistance mechanisms and perspectives of human and animal health. Microb Drug Resist 2018;24:590-606.

7. Zirakzadeh A, Patel R. Vancomycin-resistant Enterococci: Colonization, Infection, Detection, and Treatment. Mayo Clinic Proceedings. Elsevier, 2006, pp. 529-536.

8. Puchter L, Chaberny IF, Schwab F, Vonberg R-P, Bange F-C, et al. Economic burden of nosocomial infections caused by vancomycin-resistant enterococci. Antimicrob Resist Infect Control 2018;7:1-7.

9. Munoz-Price LS, Fajardo-Aquino Y, Arheart KL, Cleary T, DePascale D, et al. Aerosolization of Acinetobacter baumannii in a trauma ICU. Crit Care Med 2013;41:1915-1918.

10. Colquhoun JM, Rather PN. Insights into mechanisms of biofilm formation in Acinetobacter baumannii and implications for uropathogenesis. Front Cell Infect Microbiol 2020;10:253.

11. Lei $\mathrm{H}$, Jones RM, Li Y. Exploring surface cleaning strategies in hospital to prevent contact transmission of methicillin-resistant Staphylococcus aureus. BMC Infect Dis 2017;17:85. 
12. Spellberg B, Bonomo RA. "Airborne assault": a new dimension in Acinetobacter baumannii transmission*. Crit Care Med 2013;41:2042-2044

13. Tacconelli E, Cataldo MA. Vancomycin-resistant enterococci (VRE): transmission and control. Int J Antimicrob Agents 2008:31:99-106.

14. Dancer SJ, White LF, Lamb J, Girvan EK, Robertson C. Measuring the effect of enhanced cleaning in a UK hospital: a prospective cross-over study. BMC Medicine 2009;7:1-12.

15. Shiomori T, Miyamoto H, Makishima K. Significance of airborne transmission of methicillin-resistant Staphylococcus aureus in an otolaryngology-head and neck surgery unit. Archives of Otolaryngology-Head \& Neck Surgery 2001;127:644-648

16. Rutala WA, Weber DJ. Disinfectants used for environmental disinfection and new room decontamination technology. Am J Infect Control 2013:41:S36-41.
17. Wong T, Woznow T, Petrie M, Murzello E, Muniak A et al. Postdischarge decontamination of MRSA, VRE, and Clostridium difficile isolation rooms using 2 commercially available automated ultraviolet-C-emitting devices. Am J Infect Control 2016:44:416-420.

18. Munoz-Price LS, Weinstein RA. Acinetobacter infection. N Engl J Med 2008;358:1271-1281.

19. Rice LB. Antimicrobial resistance in gram-positive bacteria. Am J Infect Control 2006;34:S11-9;

20. Romanovskaya VA, Tashirev AB, Shilin NA, Chernaya NA Rokitko PV, et al. Resistance of Antarctic microorganisms to UV radiation. Mikrobiol Z 2011;73:3-8.

21. Arrage AA, Phelps TJ, Benoit RE, White DC. Survival of subsurface microorganisms exposed to UV radiation and hydrogen peroxide. Appl Environ Microbiol 1993;59:3545.

\section{Five reasons to publish your next article with a Microbiology Society journal}

1. The Microbiology Society is a not-for-profit organization.

2. We offer fast and rigorous peer review - average time to first decision is 4-6 weeks.

3. Our journals have a global readership with subscriptions held in research institutions around the world.

4. $80 \%$ of our authors rate our submission process as 'excellent' or 'very good'.

5. Your article will be published on an interactive journal platform with advanced metrics.

Find out more and submit your article at microbiologyresearch.org. 